\title{
Prophylactic salpingectomy in premenopausal low-risk women for ovarian cancer: Primum non nocere
}

\author{
Michele Morelli, Roberta Venturella *, Rita Mocciaro, Annalisa Di Cello, Erika Rania, Daniela Lico, \\ Pietro D'Alessandro, Fulvio Zullo \\ Department of Obstetrics \& Gynecology, “Magna Graecia” University_Gynecologic Oncology Unit, “Tommaso Campanella” Cancer Center of Germaneto, Catanzaro, Italy
}

\section{H I G H L I G H T S}

- Ovarian function is not compromised by adding bilateral salpingectomy to TLH.

- Laparoscopic bilateral salpingectomy is a safe procedure when added to TLH.

- Premenopausal prophylactic salpingectomy is a safe procedure for preventing HGSC.

\section{A R T I C L E I N F O}

\section{Article history:}

Received 11 February 2013

Accepted 23 March 2013

Available online 2 April 2013

\section{Keywords:}

High Grade Serous Ovarian Cancer

Prophylactic bilateral salpingectomy

Ovarian Reserve

Cancer prevention

\section{A B S T R A C T}

Objective. The objective of this study is to compare ovarian function and surgical outcomes between patients affected by benign uterine pathologies submitted to total laparoscopic hysterectomy (TLH) plus salpingectomy and women in which standard TLH with adnexal preservation was performed.

Methods. We retrospectively compared data of 79 patients who underwent TLH plus bilateral salpingectomy ( group A), with those of 79 women treated by standard TLH without adnexectomy (sTLH) (group B). Ovarian reserve modification, expressed as the difference between 3 months post-operative and pre-operative values of Anti-Müllerian Hormone (AMH), Follicle Stimulating Hormone (FSH), Antral Follicle Count (AFC), mean ovarian diameters and Peak Systolic Velocity (PSV), was recorded for each patient. For each surgical procedure, operative time, variation of hemoglobin level $(\Delta \mathrm{Hb})$, postoperative hospital stay, postoperative return to normal activity, and complication rate were recorded as secondary outcomes.

Results. According to our post-hoc analysis, this equivalence study resulted to have a statistical power of $96.8 \%$. Significant difference was not observed between groups with respect to $\triangle \mathrm{AMH}(p=0.35), \Delta \mathrm{FSH}(p=0.15)$, $\triangle \operatorname{AFC}(p=0.09), \Delta$ mean ovarian diameters $(p=0.57)$ and $\triangle$ PSV $(p=0.61)$. In addition, secondary outcomes such as operative time $(p=0.79), \Delta \mathrm{Hb}(p=0.41)$, postoperative hospital stay $(p=0.16)$, postoperative return to normal activity $(p=0.11$ ) and complication rate also did not show any significant difference.

Conclusions. The addition of bilateral salpingectomy to TLH for prevention of ovarian cancer in women who do not carry a BRCA1/2 mutations do not show negative effects on the ovarian function. In addition, no perioperative complications are related to the salpingectomy step in TLH.

(c) 2013 Elsevier Inc. All rights reserved.

\section{Introduction}

Ovarian cancer is still the most frequent cause of death by gynecological malignancy for women in developed countries. To date, all attempted ovarian cancer screening strategies have failed, probably because this disease represents different kinds of cancers [1]. Recently, a new classification of ovarian cancer in two different types of cancer has been introduced, where type II ovarian tumors (including

\footnotetext{
* Corresponding author at: Department of Obstetrics \& Gynaecology, "Magna Graecia" University-viale Europa, loc. Germaneto, 88100 Catanzaro, Italy. Fax: + 390961883234. E-mail address: rovefa@libero.it (R. Venturella).
}

the so called High-Grade Serous Cancer, HGSC) are considered the most frequently diagnosed, aggressive, genetically instable and often disseminated kind of disease [2].

One of the more important finding in last decade of gynecologic oncology is the confirmed theory that types II tumors derive from the epithelium of the Fallopian tube, whereas clear cell and endometrioid tumors derive from endometrial tissue that migrate to the ovary by retrograde menstruation [3].

These observations are mainly collected from women that carry BRCA1/2 mutations and underwent prophylactic salpingooophorectomy, in which most of the incidentally diagnosed in situ carcinomas or intraepithelial precursors of cancers (STIC) were detected not in the ovary but in the fimbrial end of the fallopian tube 
[4-6]. STIC were also subsequently diagnosed in many women not carrying BRCA mutations, thanks to an extended protocol of pathologic examination of the Fallopian tubes (SEE-FIM) of patients operated for sporadic HGSC [7-10].

Consequently it is probably time to ask ourselves whether it is still ethically justified not to inform our patients about the possibility of a new preventive strategy based on prophylactic salpingectomy at the time of surgery for benign pathologies.

As a matter of fact, in the general population, prophylactic salpingectomy might reduce the risk of sporadic ovarian cancer reducing at the same time the risk of premature death due to cardiovascular disease seen in women subjected to salpingo-oophorectomy before the onset of natural menopause $[11,12]$.

Bilateral salpingectomy with ovarian preservation should be considered in a cost-benefit analysis, as the best preventive strategy for women with low risk of ovarian cancer (not carrying BRCA mutations), although it is mandatory to assess the effects and complications associated with it. Nevertheless, the effects of salpingectomy on ovarian functions are still controversial. To the best of our knowledge, there are no strong evidences on the effect of salpingectomy on surgical outcomes of a standard hysterectomy.

The goal of this study is to evaluate ovarian function and surgical outcomes of patients with benign uterine pathologies that were subjected to TLH combined with bilateral salpingectomy against those who were subjected to standard TLH with adnexal preservation.

\section{Materials and methods}

\section{Patient's selection}

The procedures used in this retrospective case-control study were in accordance with the guidelines of the Helsinki Declaration on Human Experimentation and the Good Clinical Practice (CGP). The approval by the Institutional Review Board was not required due to the retrospective manner of the study. However, a written consent was obtained from all patients for the use of their clinical data prior to the beginning the study. All patients were previously instructed about the recent acknowledgment on high grade serous cancer and its suspected origin, and they signed a written consent before surgery.

All patients who underwent TLH for abnormal uterine bleeding (AUB) related to benign pathology at the Department of Obstetrics and Gynecology, Magna Graecia University of Catanzaro, between September 2010 and September 2012, were identified as group A. In all these women, ovaries were preserved but prophylactic salpingectomy was added. Data for these patients were compared with those of women treated by standard TLH (sTLH) between September 2008 and September 2010, matched for uterine weight (gr), measured after surgery (group B). Clinical and surgical data were extracted from charts and collected for each patient.

We excluded data of patients older than 50 years, with a family history of ovarian cancer, BRCA positive, basal FSH value of $>20 \mathrm{IU} / \mathrm{mL}$ and/or $E_{2}$ levels $>60 \mathrm{pg} / \mathrm{mL}$, presence of menopausal symptoms, irregular (cycle-to-cycle variation over 12 months $>20$ days or presence of any breakthrough bleeding) menstrual cycles, hormone replacement treatment and/or hormonal contraception for the last 3 months, history of previous uterine or ovarian surgeries, and imaging suggestive of ovarian cyst or tubal pathology at transvaginal ultrasound.

Patients in Group A underwent TLH and complete excision of the fallopian tubes bilaterally. Salpingectomy was performed by coagulation and section of the tube, beginning from the very distal fimbrial end, carefully preserving the ovarian vascularization, and proceeding toward the uterine cornu. Thus, fallopian tubes were removed together with the uterus. In group B, all patients had been subjected to sTLH with adnexal preservation.

\section{Clinical outcomes}

For each patient in both groups, ovarian reserve modification before and after surgery was recorded as primary outcome. In particular, according to our standard protocol, one month before and three months after laparoscopy, all patients in our Unit are usually included in a program of assessment of ovarian reserve and distance from menopause. On days 1 to 4 of menstrual cycles serum anti-Müllerian hormone $(\mathrm{AMH}, \mathrm{ng} / \mathrm{mL})$, Follicle Stimulating hormone (FSH, IU/L) and Estradiol $\left(E_{2}, \mathrm{pg} / \mathrm{mL}\right)$ were evaluated and a transvaginal ultrasound examination (Aloka ProSound SSD-3500 Ultrasound Machine-MG Medical System) to assess basal and antral follicle number [all ovarian follicles measuring $3 \mathrm{~mm}$ to $10 \mathrm{~mm}$ on the both ovaries are counted (AFC, n)], mean ovarian diameter [measured in the largest crossectional sagittal view of the ovaries, evaluating the two perpendicular diameters $(\mathrm{mm})$ ], and peak systolic velocity [measured by color Doppler ultrasound, on stromal blood vessels away from the ovarian capsule and utero-ovarian ligament, (PSV, $\mathrm{cm} / \mathrm{s}$ )] was carried out by the same experienced ultrasonographist responsible for the ambulatory of ovarian reserve.

Blood samples were obtained by venipuncture and centrifuged, within $30 \mathrm{~min}$ of collection, for $10 \mathrm{~min}$ at $4{ }^{\circ} \mathrm{C}$ at $3500 \mathrm{rpm}$, to separate the serum. Each serum sample was then stored at $-80{ }^{\circ} \mathrm{C}$ for subsequent analysis of $\mathrm{AMH}, \mathrm{FSH}$ and $\mathrm{E}_{2}$ levels. AMH Gen II Elisa assay kit (Beckman) was used to measure serum AMH levels, according to manufacturer's instruction. The lowest detection limit was $1 \mathrm{ng} / \mathrm{mL}$ and the intra and interassay coefficients of variation were respectively below $3.4 \%$ and $4.0 \%$. The ECLIA method was used to measure FSH and $\mathrm{E}_{2}$ levels in serum, using a COBAS e411 Roche auto-analyzer. The lowest detection limit for FSH was $0.1 \mathrm{IU} / \mathrm{L}$, with the intra- and interassay coefficients of variation were below $2.6 \%$ and above $3.5 \%$. The lowest detection limit for $E_{2}$ was $18.4 \mathrm{pmol} / \mathrm{L}$, with the intra- and interassay coefficients of variation were of $2 \%$ and $3 \%$, respectively.

After laparoscopic hysterectomy, due to the absence of menstrual flow, patients are instructed to identify their early follicular phase by consulting their past menstrual diary and recognizing the rapid decrease of self-reported "fluid retention" impression, indicated by a feeling of bloating, edema, and/or nicturia, in the days of the expected menses [13]. Ovarian reserve is therefore re-evaluated when early follicular phase is confirmed by the presence of serum $\mathrm{E}_{2}$ level $<60 \mathrm{pg}$ / $\mathrm{mL}$ and progesterone $(\mathrm{P})$ level $<1 \mathrm{ng} / \mathrm{mL}$, in conjunction with ultrasound evaluation (absence of a dominant follicle $>10 \mathrm{~mm}$ in any of the ovaries). Ovarian reserve modification is defined as the difference (expressed as $\Delta$ ) between post-operative and pre-operative values of $\mathrm{AMH}, \mathrm{FSH}, \mathrm{AFC}$, mean ovarian diameters and PSV.

For each surgical procedure, operative time (min), variation of hemoglobin level $(\Delta \mathrm{Hb}, \mathrm{g} / \mathrm{dL})$, postoperative hospital stay (days), postoperative return to normal activity (days), complication rate (\%) were recorded as secondary outcomes. Return to normal activity was defined as the time taken for the patient to achieve the same level of activity as to which they were capable preoperatively following discharge from hospital. This might include minor discomfort and the use of simple analgesic medication. Complete recovery was not measured because of the large variation in this non-quantitative measure. Many patients were not employed and therefore the time to return to employment was not used as an end point [14].

\section{Statistical methods}

For categorical variables, the $\chi 2$ test or Fisher exact test was applied as appropriate. The normal distribution of continuous variables was evaluated using the Kolmogrov-Smirnov test. Since continuous variables resulted in normal distribution, data were expressed as mean and standard deviation (SD). Continuous variables were analyzed using the independent samples t-test, or the paired t-test for repeated measurements as appropriate. All statistical analyses were performed 
using SPSS software, version 17.0.0 (SPSS, Inc.). P-value of $<0.05$ was used as a threshold for defining difference as statistically significant.

Post-hoc power analysis

A prior analysis conducted on our data demonstrated a post-operative AMH levels average decrease of $9 \%$ in women submitted to total laparoscopic hysterectomy with adnexal preservation (standard procedure). Assuming a 10\% decrease with this procedure, and a maximal clinically acceptable decreasing for equivalence of $15 \%$ in AMH levels in women after salpingectomy, a sample of at least 69 patients per group would have given $95 \%$ power and a one-sided significance level of $10 \%$. In our study, therefore, given a sample size of $79 \mathrm{pa}-$ tients in each group, power model resulted of $96.8 \%$.

\section{Results}

After discriminating for inclusion/exclusion criteria, we selected 79 premenopausal women treated by TLH with salpingectomy (Group A) and of other 79 patients submitted to STLH (Group B).

As detailed in Table 1, at baseline there was no significant difference between the two groups for anthropometric characteristics, obviously including uterine weight. Similarly, no statistically significant differences were observed between the two groups in $\mathrm{AMH}(0.49 \pm$ 0.4 in group $A$ vs. $0.51 \pm 0.5 \mathrm{ng} / \mathrm{mL}$ in $\mathrm{B} ; p=0.80), \mathrm{FSH}(12.3 \pm 5.2$ vs. $11.4 \pm 4.6 \mathrm{mIU} / \mathrm{mL}$ in groups $\mathrm{A}$ and $\mathrm{B}$, respectively; $p=0.27$ ), AFC ( $4.4 \pm 1.2$ vs. $4.0 \pm 2.0$ in groups $A$ and B, respectively; $p=$ $0.13)$, mean ovarian diameters $(26.7 \pm 4.1$ vs. $25.8 \pm 3.8 \mathrm{~mm}$ in groups $\mathrm{A}$ and $\mathrm{B}$, respectively; $p=0.16)$ and PSV (10.1 \pm 2.1 vs. $9.5 \pm 2.0 \mathrm{~cm} / \mathrm{s}$ in groups $\mathrm{A}$ and $\mathrm{B}$, respectively; $p=0.07$ ). $\mathrm{E}_{2}$ levels were statistically significant between the two groups, but these values were not clinically relevant, because they were always lower than $60 \mathrm{pg} / \mathrm{mL}$.

Table 2 shows the primary outcome measures. As detailed in the materials and methods, the ovarian reserve modification is expressed as the $\Delta$ between post-operative and pre-operative values of AMH, FSH, AFC, mean ovarian diameters and PSV. Statistically or clinically significant difference was not observed between groups for parameters evaluating the ovarian reserve after surgery. Both groups were homogeneous for $\triangle \mathrm{AMH}(-0.06 \pm 0.1$ vs. $-0.08 \pm$ $0.1 \mathrm{ng} / \mathrm{mL}$ in groups A and B, respectively; $p=0.35), \Delta \mathrm{FSH}(1.3 \pm$ 1.1 vs. $1.0 \pm 0.8 \mathrm{mIU} / \mathrm{mL}$ in groups $\mathrm{A}$ and $\mathrm{B}$, respectively; $p=$ $0.15), \Delta \mathrm{AFC}(-0.27 \pm 0.6$ vs. $-0.14 \pm 0.3$ in groups $\mathrm{A}$ and $\mathrm{B}$, respectively; $p=0.09), \Delta$ mean ovarian diameters $(-0.25 \pm 0.8 v s$. $-0.19 \pm 0.6 \mathrm{~mm}$ in groups $\mathrm{A}$ and $\mathrm{B}$, respectively; $p=0.57$ ) and $\triangle \mathrm{PSV}(-0.31 \pm 1.9$ vs. $-0.19 \pm 1.0 \mathrm{~cm} / \mathrm{s}$ in groups $\mathrm{A}$ and $\mathrm{B}$, respectively; $p=0.61)$.

Regarding the secondary outcomes, there was no statistically nor clinically significant difference between groups A and B for operative time, $\Delta \mathrm{Hb}$, postoperative hospital stay, postoperative return to normal activity and complication rate (\%). Data is provided in Table 3.

Table 1

Main anthropometric and hormonal measures at baseline.

\begin{tabular}{lccc}
\hline Parameters & $\begin{array}{c}\text { TLH plus salpingectomy } \\
\text { Group A (n.79) }\end{array}$ & $\begin{array}{c}\text { Standard TLH } \\
\text { Group B (N.79) }\end{array}$ & $p$ \\
\hline Age $($ years $)$ & $45.8 \pm 2.4$ & $46.5 \pm 2.9$ & 0.10 \\
BMI $\left(\mathrm{kg} / \mathrm{m}^{2}\right)$ & $22.8 \pm 1.8$ & $23.3 \pm 1.7$ & 0.13 \\
Uterine weight $(\mathrm{g})$ & $328.9 \pm 115.0$ & $316.9 \pm 149.0$ & 0.62 \\
AMH $(\mathrm{ng} / \mathrm{mL})$ & $0.49 \pm 0.4$ & $0.51 \pm 0.5$ & 0.80 \\
FSH $(\mathrm{mIU} / \mathrm{mL})$ & $12.3 \pm 5.2$ & $11.4 \pm 4.6$ & 0.27 \\
E $_{2}(\mathrm{pg} / \mathrm{mL})$ & $22.8 \pm 15.9$ & $28.1 \pm 15.1$ & 0.04 \\
AFC $(\mathrm{n})$ & $4.4 \pm 1.2$ & $4.0 \pm 2.0$ & 0.13 \\
Mean ovarian diameters $(\mathrm{mm})$ & $26.7 \pm 4.1$ & $25.8 \pm 3.8$ & 0.16 \\
PSV $(\mathrm{cm} / \mathrm{s})$ & $10.1 \pm 2.1$ & $9.5 \pm 2.0$ & 0.07 \\
\hline
\end{tabular}

All data are expressed as mean and SD.
Table 2

Primary outcomes measures.

\begin{tabular}{llcr}
\hline Parameters & $\begin{array}{l}\text { TLH plus salpingectomy } \\
\text { Group A (n.79) }\end{array}$ & $\begin{array}{l}\text { Standard TLH } \\
\text { Group B (N.79) }\end{array}$ & $p$ \\
\hline$\Delta$ AMH $(n g / m L)$ & $-0.06 \pm 0.1$ & $-0.08 \pm 0.1$ & 0.35 \\
$\Delta$ FSH $(\mathrm{mIU} / \mathrm{ml})$ & $1.3 \pm 1.1$ & $1.0 \pm 0.8$ & 0.15 \\
$\Delta$ AFC $(n)$ & $-0.27 \pm 0.6$ & $-0.14 \pm 0.3$ & 0.09 \\
$\Delta$ Mean ovarian diameters $(\mathrm{mm})$ & $-0.25 \pm 0.8$ & $-0.19 \pm 0.6$ & 0.57 \\
$\Delta$ PSV $(\mathrm{cm} / \mathrm{s})$ & $-0.31 \pm 1.9$ & $-0.19 \pm 1.0$ & 0.61 \\
\hline
\end{tabular}

All data are expressed as mean and SD.

\section{Discussion}

In this study we demonstrate that ovarian function and surgical outcomes in patients submitted to TLH for benign uterine pathologies are not modified by the addiction of bilateral salpingectomy to the standard technique. Both hormonal and ultrasonographic parameters, indeed, are not significantly altered when salpingectomy is performed together with our surgical standard technique.

Our surgical technique carefully preserves the ovarian blood supply, ensured by an arcade-like anastomosis system formed by ovarian artery and the tubal branch of the uterine artery at the junction of the mesosalpinx and the mesovary, close to the hilum of the ovary. The attention to blood supply when adding bilateral salpingectomy to TLH may explain our positive results in terms of post-operative ovarian function, since an intact blood flow is essential to a normal ovarian steroid hormone synthesis.

Preservation of the ovarian function is important both in the pre-menopausal age and in the post-menopause, due to the effective prevention of bone resorption, guaranteed by the intact ovaries [15-17]. Furthermore, surgical menopause increases long-term risk of psychosexual, cognitive and cardiovascular dysfunctions [18-20] and incidence of fatal and non-fatal coronary heart diseases [21].

These data suggest that, at least for premenopausal women with no genetic risk for ovarian cancer, oophorectomy at the time of hysterectomy should be approached with great caution $[21,22]$.

Considering the new acquisitions in the field of HGSC, our improved understanding of its pathogenesis surely opens new opportunities for prevention, in which every gynecologic surgeons, not only oncologist, can play an important role by performing bilateral salpingectomy during any kind of hysterectomy (laparoscopic, laparotomic or vaginal) or at the time of surgical sterilization [23].

The preservation of the Fallopian tubes during hysterectomy is a surgical strategy with no known benefits and conversely, the blind-ended remnants may give rise to complications, such as hydrosalpinx [24,25], which often requires recurring surgeries.

Thus, removal of the tubes rather than the ovaries could be efficient in preventing HGSC, potentially avoiding issues of long-term surgical complication and hormonal function.

According to the existing literature, however, whether bilateral salpingectomy impairs ovarian reserve is still a matter of debate. Animal studies shows that there is no impairment [25-27] albeit there are

Table 3

Secondary outcomes measures.

\begin{tabular}{llll}
\hline Parameters & $\begin{array}{l}\text { TLH plus salpingectomy } \\
\text { Group A (n.79) }\end{array}$ & $\begin{array}{l}\text { Standard TLH } \\
\text { Group B (N.79) }\end{array}$ & $p$ \\
\hline Operative time $(\mathrm{min})$ & $81.7 \pm 14.8$ & $83.3 \pm 18.6$ & 0.79 \\
$\Delta \mathrm{Hb}(\mathrm{g} / \mathrm{dL})$ & $1.1 \pm 0.9$ & $1.2 \pm 0.7$ & 0.41 \\
$\begin{array}{l}\text { Postoperative hospital stay } \\
\quad \text { (days) }\end{array}$ & $2.5 \pm 0.8$ & $2.7 \pm 0.8$ & 0.16 \\
$\begin{array}{l}\text { Postoperative return to normal } \\
\quad \text { activity (days) }\end{array}$ & $15.0 \pm 4.4$ & $13.9 \pm 4.8$ & 0.11 \\
Complication rate $(\%)$ & 0 & 0 & - \\
\hline
\end{tabular}

All data are expressed as mean and SD. 
conflicting results on ovarian response to medical induction of superovulation in patients treated by salpingectomy $[28,29]$.

Recently, Sezik Mekin et al. investigated the effect of total salpingectomy during hysterectomy in a small sample of 12 patients compared to other 12 treated by partially salpingectomy. In their patients, hormonal parameters were unchanged after both strategies, but ovarian stromal blood flow appeared to be decreased in both groups compared to baseline values [30]. The small statistical power of the investigation, however, limited the reliability of their data.

To the best of our knowledge, the present study is the first comprehensive comparison of the outcomes of TLH with and without bilateral salpingectomy, evaluating the ovarian function and surgical parameters in a large population of patients. Despite the retrospective fashion of the study design, according to our post-hoc analysis, we obtained a power model of $96.8 \%$. This result allows us to ensure our data with a good statistical reliability.

Our demonstration of the safety of adding bilateral salpingectomy to TLH can be the first step to declaring that the new proposed strategy to prevent ovarian cancer does not cause harm to our patients, allowing us to respect our first medical rule, primum non nocere. Although the new data on the pathogenesis of ovarian cancer cannot be considered $100 \%$ verified, we wonder if it is ethically justified not to counsel our patients about the possibility of a simple preventive strategy, such as salpingectomy [31].

Considering that in developed countries hysterectomy is the second most frequently performed surgical intervention among women of reproductive age, and in USA about one-third of all 60-year-old women have had a hysterectomy [32], it is clear that a strong rational exists to justify the British Columbia Ovarian Cancer Prevention Project. It encourages prophylactic salpingectomy and estimates up to $50 \%$ reduction in ovarian cancer deaths after 20 years, up to $20 \%$ through salpingectomy at time of hysterectomy, up to $20 \%$ through salpingectomy instead of tubal ligation and up to $20 \%$ through risk-reducing bilateral salpingo-oophorectomy in patients with BRCA mutations [33].

Moreover, by extending the practice of performing SEE-FIM [10] in all salpingectomy specimens, it will be possible to provide a definitive population-based assessment of the frequency and risk of STIC in risk-reducing specimens in low risk women with BRCA-negative family background.

Another important short term objective will be to carefully characterize the histopathological and molecular features of the precursor lesions found in the fallopian tubes, to enable us to find the diagnostic tools for defining a "medium risk" category of women (without the BRCA mutations) in which we hypothesize either a preventive bilateral salpingectomy once they have accomplished their reproductive desire or a medical chemoprevention.

In conclusion, our demonstration that ovarian function and surgical outcomes in patients submitted to TLH are not modified by adding salpingectomy to the standard technique is the first answer to the discordance about the degree of influence on ovarian reserve related to tubal surgery. We demonstrate that if salpingectomy is performed with great care by preserving blood vessels integrity in the proximity of the ovarian hilum and in the context of the mesosalpinx, patients will not have negative effects in on their ovarian function. In addition, in our experience, no perioperative complications associated with the procedure are attributable to salpingectomy alone. Salpingectomy can be defined as a simple and safe surgical procedure, not harmful for our patients, in terms of ovarian function and surgical outcomes.

\section{Conflict of interest statement}

All the authors declare that they have no competing interests.

\section{Acknowledgment}

The authors acknowledge Prof. Rita Colacino for her helpful assistance on language editing.

\section{References}

[1] Chan A, Gilks B, Kwon J, Tinker AV. New insights into the pathogenesis of ovarian carcinoma. Time to rethink ovarian cancer screening. Obstet Gynecol 2012;120:935-40.

[2] Crum CP, Drapkin R, Miron A, et al. The distal fallopian tube: a new model for pelvic serous carcinogenesis. Curr Opin Obstet Gynecol 2007;19:3-9.

[3] Kurman RJ, Shih Ie M. The origin and pathogenesis of epithelial ovarian cancer: a proposed unifying theory. Am J Surg Pathol 2010;34:433-43.

[4] Crum CP, Drapkin R, Kindelberger D, Medeiros F, Miron A, Lee Y. Lessons from BRCA: the tubal fimbria emerges as an origin for pelvic serous cancer. Clin Med Res 2007:5:35-44.

[5] Manchanda R, Abdelraheim A, Johnson M, Rosenthal A, Benjamin E, Brunell C, et al. Outcome of risk-reducing salpingo-oophorectomy in BRCA carriers and women of unknown mutation status. BJOG 2011;118:814-24.

[6] Powell CB, Chen LM, McLennan J, Crawford B, Zaloudek C, Rabban JT, et al. Risk-reducing salpingo-oophorectomy (RRSO) in BRCA mutation carriers: experience with a consecutive series of 111 patients using a standardized surgical pathological protocol. Int J Gynecol Cancer 2011;21:846-51.

[7] Crum CP, McKeon FD, Xian W. BRCA, the oviduct, and the space and time continuum of pelvic serous carcinogenesis. Int J Gynecol Cancer 2012;22:S29-34.

[8] Salvador S, Rempel A, Soslow RA, Gilks B, Hunsman D, Miller D. Chromosomal instability in fallopian tube precursor lesions of serous carcinoma and frequent monoclonality of synchronous ovarian and fallopian tube mucosal serous carcinoma. Gynecol Oncol 2008;110:408-17.

[9] Wiegand KC, Shah SP, Al-Agha OM, Zhao Y, Tse K, Zeng T, et al. ARID1A mutations in endometriosis-associated ovarian carcinomas. N Engl J Med 2010;363:1532-43.

[10] Medeiros F, Muto MG, Lee Y, et al. The tubal fimbria is a preferred site for early adenocarcinoma in women with familial ovarian cancer syndrome. Am J Surg Pathol 2006;30:230-6.

[11] Kwon JS, Tinker A, Pansegrau G, McAlpine J, Housty M, McCullum M, et al. Prophylactic salpingectomy and delayed oophorectomy as an alternative for BRCA mutation carriers. Obstet Gynecol 2012:1-11.

[12] Parker WH, Broder MS, Chang E, Feskanich D, Farquhar C, Liu Z, et al. Ovarian conservation at the time of hysterectomy and long-term health outcomes in the nurses' health study. Obstet Gynecol 2009;113:1027-37.

[13] White CP. Hitchcock CL, Vigna YM, Prior JC. Fluid retention over the menstrual cycle: 1-year data from the prospective ovulation cohort. Obstet Gynecol Int 2011;2011:138451, doi:10.1155/2011/138451.

[14] Raymond TM, Dastur JK, Khot UP, Parker MC. Hospital stay and return to full activity following laparoscopic colorectal surgery. JSLS 2008;12:143-9.

[15] Judd HL, Judd GE, Lucas WE, Yen SS. Endocrine function of the postmenopausal ovary: concentration of androgens and estrogens in ovarian and peripheral vein blood. J Clin Endocrinol Metab 1974;39:1020-4.

[16] Fogle RH, Stanczyk FZ, Zhang X, Paulson RJ. Ovarian androgen production in postmenopausal women. J Clin Endocrinol Metab 2007;92:3040-3.

[17] Melton III LJ, Khosla S, Malkasian GD, Achenbach SJ, Oberg AL, Riggs BL. Fracture risk after bilateral oophorectomy in elderly women. J Bone Miner Res 2003 May:18:900-5.

[18] Falkeborn M, Schairer C, Naessen T, Persson I. Risk of myocardial infarction after oophorectomy and hysterectomy. J Clin Epidemiol 2000;53:832-7.

[19] Rocca WA, Bower JH, Maraganore DM, Ahlskog JE, Grossardt BR, de Andrade M, et al Increased risk of cognitive impairment or dementia in women who underwent oophorectomy before menopause. Neurology 2007;69:1074-83.

[20] Rivera CM, Grossardt BR, Rhodes DJ, Brown Jr RD, Roger VL, Melton III LJ, et al. Increased cardiovascular mortality after early bilateral oophorectomy. Menopause 2009;16:15-23.

[21] Parker WH, Jacoby V, Shoupe D, Rocca W. Effect of bilateral oophorectomy on women's long-term health. Womens Health (Lond Engl) 2009:5:565-76.

[22] ACOG Practice Bulletin No. 89. Elective and risk-reducing salpingo oophorectomy. Obstet Gynecol 2008;111:231-41.

[23] Dietl J, Wischhusen J, Häusler SF. The post-reproductive fallopian tube: better removed? Hum Reprod 2011;26:2918-24, doi:10.1093/humrep/der274.

[24] Morse AN, Hammer RA, Walter AJ, Baker S, Magtibay PM. Does hysterectomy without adnexectomy in patients with prior tubal interruption increase the risk of subsequent hydrosalpinx? Am J Obstet Gynecol 2002;187:1483-6.

[25] Repasy I, Lendvai V, Koppan A, Bodis J, Koppan M. Effect of the removal of the fallopian tube during hysterectomy on ovarian survival: the orphan ovary syndrome. Eur J Obstet Gynecol Reprod Biol 2009;144:64-7.

[26] Halme J, Rong ZJ, Wing R, Raj MH, Raj S. The removal of fallopian tubes has no adverse effect of subsequent ovarian function in rabbits. Fertil Steril 1982;38:621-5.

[27] Zhao JR, Wing R, Hulka JF. Ovarian function in monkeys after bilateral salpingoectomy. Int J Fertil 1984;29:118-21.

[28] Strandell A, Lindhard A, Waldenström U, Thorburn J. Prophylactic salpingectomy does not impair the ovarian response in IVF treatment. Hum Reprod 2001 Jun;16:1135-9.

[29] Chan CC, Ng EH, Li CF, Ho PC. Impaired ovarian blood flow and reduced antral follicle count following laparoscopic salpingectomy for ectopic pregnancy. Hum Reprod 2003;18:2175-80.

[30] Sezik M, Ozkaya O, Demir F, Sezik HT, Kaya H. Total salpingectomy during abdominal hysterectomy: effects on ovarian reserve and ovarian stromal blood flow. J Obstet Gynaecol Res 2007;33:863-9.

[31] Westgren M. Prevention of ovarian cancer-let's do something. Acta Obstet Gynecol Scand 2012;91:1009-10.

[32] Whiteman MK, Hillis SD, Jamieson DJ, Morrow B, Podgornik MN, Brett KM, et al. Inpatient hysterectomy surveillance in the United States, 2000-2004.Am J Obstet Gynecol 2008;198:34 [e31-37].

[33] http://www.ovcare.ca. 